\title{
LETTER
}

\section{Usefulness and limitations of single-slice computed tomography analysis at the third lumbar region in the assessment of sarcopenia}

\author{
Vildan Binay Safer ${ }^{1 *}$ and Umut Safer ${ }^{2}$ \\ See related research by Moisey et al., http://ccforum.com/content/17/5/R206
}

In the previous issue of Critical Care, Moisey and colleagues [1] report that sarcopenia has potential to serve as a predictor for mortality, discharge disposition, and ICU utilization. Sarcopenia may be mitigated by adequate nutrition supply and early rehabilitation, and so identification of patients with ICU-acquired weakness, who could benefit from rehabilitation, is of fundamental importance [2]. and this limited the generalization of results for the ICU setting.

The EWGSOP recommends the measurement of muscle power, function, and mass for a sarcopenia diagnosis [3]. As muscle function and power data of patients were not reported in the present article, we do not know whether all participants were accurately classified as sarcopenic.

\section{Authors' response \\ Marina Mourtzakis, Lesley Moisey, Daren Heyland and Rosemary Kozar. We appreciate the interest of Binay Safer and Safer in our article.}

Sarcopenia diagnosis is based on estimation of muscle mass. Whole-body imaging methods like computed tomography (CT) and magnetic resonance imaging (MRI) can precisely measure muscle mass, but limitations of these modalities led to new tools for daily practice. The European Working Group on Sarcopenia in Older People (EWGSOP) recommends the use of dual-energy $x$-ray absorptiometry (DXA) as an alternative [3]. Nowadays, single-slice CT image analysis, which was used in the study by Moisey and colleagues, is becoming a popular alternative. Validation of this technique was previously assessed by comparing the single-slice CT analysis at the third lumbar (L3) region with DXA, which is not the gold standard [4]. Measurement errors related to DXA might affect these validation results. Although Shen and colleagues [5] compared single-slice CT analysis and whole-body MRI, they reported results for the fourth-fifth lumbar region, not for the L3 region. Moreover, this study was conducted among healthy subjects

\footnotetext{
* Correspondence: binayvildan@yahoo.com

'Department of Physical Medicine and Rehabilitation, Ankara Physical Medicine Rehabilitation Research and Training Hospital, Turkocagi Street, 06230, Ankara, Turkey

Full list of author information is available at the end of the article
}

\section{Definition of sarcopenia}

The EWGSOP recommends that sarcopenia be defined as low muscularity as well as loss of muscle strength or performance or both [1], necessitating advances in precisely measuring muscle mass. The authors correctly indicate that we did not measure muscle strength/ performance. However, our study was performed on critically injured, older trauma patients at the time of admission, prohibiting any meaningful assessment of muscle strength. We present a novel approach that is highly accessible and practical as CT scans are frequently performed as part of routine care for the injured patient. Our aim goes beyond categorizing sarcopenic versus non-sarcopenic patients to show differences in various clinical outcomes (note that muscle, as a continuous variable, is also associated with mortality) [2]. Our ultimate goal is to identify vulnerable patients who require specific nutritional or rehabilitative intervention and assess success or failure of this intervention by using CT. $\mathrm{CT}$ imaging is one of a few modalities that measure muscle specifically and is far superior to body weight (most commonly used to measure nutritional status in the ICU). Single-slice CT images in the lumbar region (including L3) have been strongly associated with whole- 
body muscle volume by using MRI in healthy people [3] and DXA in a clinical population [4], indicating that lumbar muscle is representative of whole-body muscle.

\section{Computed tomography versus dual-energy $\mathrm{x}$-ray absorptiometry}

When CT and MRI are not available, DXA is recommended by the EWGSOP as an alternative method. DXA is not always available, accessible, practical, or feasible for critically injured patients. Transporting these patients for additional studies carries risk and does incur additional cost. Importantly, DXA measures lean body mass, which includes skeletal muscle as well as internal organs such as liver and spleen that may be confounding during critical illness. Isolating appendicular muscle is possible but requires some manual analysis and may introduce human error.

\section{Abbreviations}

CT: Computed tomography; DXA: Dual-energy x-ray absorptiometry; EWGSOP: European Working Group on Sarcopenia in Older People; L3: Third lumbar; MRI: Magnetic resonance imaging.

\section{Competing interests}

The authors declare that they have no competing interests.

\section{Author details}

'Department of Physical Medicine and Rehabilitation, Ankara Physical Medicine Rehabilitation Research and Training Hospital, Turkocagi Street, 06230, Ankara, Turkey. ${ }^{2}$ Department of Geriatrics, Gulhane School of Medicine, General Doktor Tevfik Saglam Street, 06018, Ankara, Turkey.

\section{Published: 20 Nov 2013}

\section{References}

1. Moisey LL, Mourtzakis M, Cotton BA, Premji T, Heyland DK, Wade CE, Bulger E, Kozar RA: Skeletal muscle predicts ventilator-free days, ICU-free days, and mortality in elderly ICU patients. Crit Care 2013, 17:R206.

2. Puthucheary Z, Hart N: Intensive care unit acquired muscle weakness: when should we consider rehabilitation? Crit Care 2009, 13:167.

3. Cruz-Jentoft AJ, Baeyens JP, Bauer JM, Boirie Y, Cederholm T, Landi F, Martin FC, Michel J-P, Rolland Y, Schneider SM, Topinková E, Vandewoude M, Zamboni M: Sarcopenia: European consensus on definition and diagnosis: Report of the European Working Group on Sarcopenia in Older People. Age Ageing 2010, 39:412-423.

4. Mourtzakis M, Prado CM, Lieffers JR, Reiman T, McCargar L, Baracos VE: A practical and precise approach to quantification of body composition in cancer patients using computed tomography images acquired during routine care. Appl Physiol Nutr Metab 2008, 33:997-1006.

5. Shen W, Punyanitya M, Wang Z, Gallagher D, St-Onge M-P, Albu J, Heymsfield SB, Heshka S: Total body skeletal muscle and adipose tissue volumes: estimation from a single abdominal cross-sectional image. J Appl Physiol 2004, 97:2333-2338.

\section{$10.1186 / \mathrm{cc} 13123$}

Cite this article as: Binay Safer and Safer: Usefulness and limitations of single-slice computed tomography analysis at the third

lumbar region in the assessment of sarcopenia. Critical Care 2013, 17:466 